\title{
Les unités de traduction existent-elles dans la chanson traduite?
}

\author{
Giulia D'Andrea*
}

\section{Introduction}

La traduction chantée est traditionnellement négligée dans le cadre des études sur la traduction, ce que déplorent aussi bien les chercheurs que les professionnels. Ce sujet pose néanmoins des questions intéressantes par son caractère interdisciplinaire, notamment en raison des défis liés à l'interface langue-musique. Il est bien connu que les auteurs des traductions chantées ont plutôt une formation de paroliers ; par ailleurs, l'intérêt suscité par la traduction chantée semble aller au-delà du domaine strictement artistique, comme le montre Jean-René Ladmiral (2004). En partant du problème apparemment marginal de la dimension musicale des livrets d'opéra, il élabore un modèle théorique plus général, dans lequel ce type tout particulier de traduction peut être considéré comme un paradigme pour 1'“Esthétique de la traduction” telle qu'il l'entend (Ladmiral 2004, p. 32).

Dans la présente étude, nous nous interrogeons sur la pertinence de la notion d'unité de traduction dans le cadre de la traduction chantée et plus spécifiquement dans celui de la chanson. Après avoir défini ce que nous

\footnotetext{
${ }^{*}$ Université du Salento (Lecce - Italie).
} 
entendons par "traduction chantée", nous évoquerons quelques contributions significatives en la matière, pour nous pencher ensuite sur la notion d'unité de traduction et sur l'objet d'étude chanson. En croisant ces deux notions, nous essayerons de déceler s'il est possible d'identifier des unités de traduction chantée.

\section{Sur la traduction chantée : essai de définition}

Les livrets d'opéra et les paroles de chansons semblent être les textes chantés les plus étudiés d'un point de vue traductologique : dans les deux cas, on constate aussi bien la présence de traductions effectuées pour faciliter la compréhension des textes de départ, que celle de véritables traductions chantées.

Pour l'opéra, partant d'un cas d'étude spécifique, Céline Frigau (2011) met bien en évidence les différences sémiotiques et stylistiques de ces différents types de traduction, ainsi que les implications socioculturelles liées au contexte de production et de réception de la traduction de la part du public. Pour la chanson, il convient aussi de mentionner deux techniques de pseudo-traduction adoptées à partir des années 1930, à savoir la "traduzione da titolo" et la "traduzione ad orecchio" (Sacchi 2003, p. 10-13). Dans le premier cas, on se contente de réécrire des chansons dans une autre langue en ne se souciant que de la fidélité sémantique au titre de l'original ; dans le second cas, on joue sur le pouvoir évocateur de termes étrangers, notamment espagnols, dont la similitude phonétique avec l'italien contribue à la réception de la version en langue d'arrivée. Ce sont deux stratégies de réécriture qui témoignent d'une tendance à considérer les paroles des chansons comme interchangeables. 
Pour résumer, dans le cadre de la traduction de textes chantés, il convient de bien distinguer les traductions à lire, réalisées dans le but de faire comprendre la signification des textes, et les traductions destinées à être elles-mêmes chantées. Ce n'est que ce deuxième type de traduction que nous appelons "traduction chantée". Autrement dit, par "traduction chantée" nous nous référons à une traduction interlinguistique où le texte de départ et le texte d'arrivée sont non seulement équivalents du point de vue sémantique et pragmatique, mais aussi chantés plus ou moins sur la même musique1.

La littérature scientifique sur la traduction chantée proprement dite, bien que restreinte, comprend des écrits portant sur des aspects variés et pouvant être classés selon plusieurs critères, comme les genres musicaux. Les livrets d'opéra représentent un des domaines les plus étudiés en France²: si aujourd'hui on a tendance à surtitrer les opéras étrangers, il n'en a pas toujours été ainsi. Au 19e siècle, par exemple, la traduction en français d'opéras italiens ou allemands était largement pratiquée dans un but pragmatique, mais elle ne faisait pas l'objet d'une réflexion théorique de la part des traducteurs-adaptateurs, ni de la critique (Schneider 2002). Dans un tel contexte, et bien avant que la traductologie ne s'établisse comme discipline autonome, Castil-Blaze (1784-1857) fait figure d'exception car il consacre beaucoup de pages à des observations ponctuelles et intéressantes concernant la traduction d'opéras (Castil-Blaze 1820 ; 1858).

Les recherches sur la traduction chantée peuvent être présentées non seulement à partir du genre musical auquel appartient le texte chanté, mais aussi

\footnotetext{
${ }^{1}$ Nous reviendrons sur ce point au $\S 4$.

${ }^{2}$ Cf. notamment le riche volume dirigé par Marschall (2004).
} 
en fonction des différentes approches adoptées ${ }^{3}$. Par exemple, on peut y trouver des discussions sur l'opportunité de traduire ou non un opéra, ou des réflexions sur les implications socioculturelles liées au chant comme pratique identitaire. Notre point de vue étant prioritairement linguistique, nous privilégions les études qui cherchent à décrire cette composante des traductions chantées existantes. En particulier, nous nous focalisons sur le genre de la chanson. Nos références bibliographiques, qui ne se veulent pas exhaustives, sont justement sélectionnées dans le but de témoigner de ce choix.

Les études linguistiques sur la traduction des chansons semblent s'être développées plus en Italie qu'en France. En témoigne surtout le volume issu d'un Colloque scientifique consacré à la traduction de la chanson d'auteur (Garzone-Schena 2000) et dont les contributions se focalisent sur des aspects essentiels de la question, comme la "cantabilità" du texte traduit (Menin 2000, p. 55-56) ou la contrainte sémiotique qui caractérise l'opération traduisante (Garzone 2000, p. 78). Les auteurs de ce volume se concentrent aussi sur la distinction fondamentale entre la traduction chantée et les cover/reprises chantées sur la même musique mais n'ayant presque pas de lien sémantique avec le texte de départ - (Garzone 2000, p. 99), ainsi que sur les non moins importantes différences épistémologiques entre traduction interlinguistique et traduction endosémiotique (Salmon 2000, p. 117-118).

En ce qui concerne les chercheurs italiens, l'œuvre de Mirella Conenna mérite une mention à part : en partant du cas exemplaire des traductions des chansons de Georges Brassens, auquel elle a consacré plusieurs articles ainsi que

\footnotetext{
${ }^{3}$ Pour une vue d'ensemble sur les liens entre traduction et musique et sur les possibles pistes de recherches $\mathrm{v}$. notamment Susam-Sarajeva (2008).
} 
les actes d'un colloque (Conenna 1998), elle a contribué à développer la réflexion autour de la traduction de l'objet-chanson. Son approche se focalise sur la dimension poétique des textes et sur la composante linguistique de leurs traductions; néanmoins, elle est consciente que les contraintes métriques et musicales représentent le point névralgique de toute traduction chantée. La chanson traduite a tendance à être reçue comme un produit original, ce qui constitue une autre de ses spécificités : en effet, même si la musique la lie à sa version originale, elle peut vivre d'une vie autonome. Ceci en particulier grâce au rôle joué par le traducteur-interprète, qui peut être identifié comme auteur (Conenna 2000, p. 155).

Parmi les études italiennes consacrées aux chansons traduites, nous tenons à mentionner un petit volume intitulé La Tradotta. Storie di canzoni amate e tradite (De Angelis ; Sacchi 2003). Le titre de ce livre joue sur la polysémie du mot italien "tradotta", à la fois participe passé du verbe "tradurre" et substantif renvoyant à un type de train destiné au transport des troupes militaires : y sont réunies les contributions de professionnels ayant une expérience directe en matière de chansons traduites. Il s'agit notamment d'auteurs-compositeursinterprètes et de paroliers qui focalisent leur attention sur des aspects bien plus concrets et ponctuels que ceux généralement traités par les chercheurs, comme la question des droits d'auteur, les politiques commerciales adoptées par les maisons discographiques et les limites imposées par la censure.

Si l'on se focalise sur la langue italienne comme langue d'arrivée, les chercheurs et les traducteurs de chansons insistent sur un point: parmi les problèmes techniques de la traduction chantée, il y a surtout la difficulté à récréer des vers oxytons dans une langue italienne où la plupart des mots sont 
paroxytons. Quant à la traduction, une de nos études (D'Andrea 2014) illustre quelques-unes des principales stratégies adoptées pour essayer de résoudre ce problème : dans une perspective où "l'activité de traduction d'une chanson est comparée à la composition d'une chanson au lieu d'être considérée dans ses rapports avec la traduction des textes poétiques" (p. 80), nous avons mentionné l'emploi de mots oxytons en soi (tel "perché") ou qui sont traités comme oxytons, qu'il s'agisse de monosyllabes (tels les mots-chevilles " $m e^{\prime \prime}$, "te", etc.) ou de certaines voix verbales se terminant par une diphtongue descendante (tels "sai", "dimenticherai", etc.) ; la taxonomie élaborée comprend également des procédés comme la troncation de mots paroxytons (tel "mattin" au lieu de "mattino") ou la transformation de mots proparoxytons en des mots oxytons, fondée sur une sorte de double accentuation (tel "secoli"). Une mention à part mérite le caslimite de certains dialectes gallo-romans, parfois choisis comme langues d'arrivée au lieu de l'italien par certains auteurs-compositeurs-interprètes de renom, tels Fausto Amodei ou Nanni Svampa, pour traduire les chansons de Georges Brassens.

En ce qui concerne l'anglais langue cible, il convient ici de mentionner l'approche originale élaborée par Peter Low (2005) à partir de la théorie du skopos de Vermeer (1978; 2000), et appelée “Principe du pentathlon". Low énumère cinq critères censés guider le travail du traducteur de chansons, à savoir la chantabilité, le sens, l'idiomaticité, le rythme et la rime. Pour la traduction de l'opéra, un sixième critère est requis, l'efficacité dramatique ("hexathlon"). Dans la perspective de Low, le traducteur de textes chantés devrait avoir la même attitude flexible qu'un athlète dont la stratégie consiste à doser les efforts 
afin d'optimiser le score d'ensemble et non pas d'atteindre le score le plus élevé dans chaque discipline.

Comme nous le montrerons dans les paragraphes qui suivent, la flexibilité joue aussi un rôle essentiel dans l'identification des unités de traduction chantée.

\section{Les unités de traduction et la traduction chantée}

À notre connaissance, la plupart des études sur la traduction chantée n'abordent pas explicitement la notion d'unité de traduction. Néanmoins, il s'agit d'une question cruciale tant pour les auteurs des traductions chantées que pour ceux qui cherchent à les décrire : les premiers s'en servent au cours de leur activité traduisante, les autres en ont besoin pour mieux formuler leurs réflexions traductologiques.

Nous nous penchons dans cette section sur l'unité de traduction dorénavant "UT" - dans le double objectif de rappeler comment est né ce terme et d'en comprendre la signification. Il s'agit, par ailleurs, d'une notion qui a fait l'objet de plusieurs débats et qui a suivi une certaine évolution en traductologie. Nous ne pourrons qu'en rendre compte partiellement, le but majeur de cette étude étant d'apporter quelques éléments de réflexion à propos de la pertinence de cette notion pour la traduction chantée, et notamment pour le genre de la chanson ${ }^{4}$.

\footnotetext{
${ }^{4}$ Pour une vue d'ensemble sur ce sujet, cf. le numéro 45 de la revue Équivalences, intitulé Des unités de traduction à l'unité de la traduction, coordonné par Nicolas Froeliger, Lance Hewson et Christian Balliu.
} 


\subsection{Les UT : une prémisse conceptuelle}

En traductologie, on a coutume d'attribuer la paternité du concept d'unité de traduction à Vinay et Darbelnet (1958, p. 37), d'après qui elle serait "le plus petit segment de l'énoncé dont la cohésion des signes est telle qu'ils ne doivent pas être traduits séparément". Cette définition, élaborée dans le cadre de leur stylistique comparée, a notamment pour but de souligner combien le mot s'avère ne pas être l'unité la plus adéquate en traduction, parce que "avec lui on ne voit plus clairement la structure double du signe et que le signifiant prend une place exagérée par rapport au signifié". Le traducteur devant traduire des idées et non des mots, Vinay et Darbelnet (1958, p. 37-38) défendent l'idée d'une unité de pensée, qui peut se manifester sous forme d'unité sémantique, dialectique ou prosodique et, dans certains cas, fonctionnelle.

La définition formulée dans le cadre de la stylistique comparée a fait l'objet de nombreuses critiques, dont celle élaborée par Michel Ballard (2006) à propos des procédés de traduction. Au lieu d'un concept d'UT centré sur le prédécoupage du texte de départ, Ballard propose de redéfinir l’UT par rapport à un modèle triadique de la traduction caractérisé non seulement par une recherche de l'équivalence, mais aussi par une phase herméneutique et par une phase de réécriture. D’après Ballard (2006 § 43), la configuration de l’UT serait donc variable en fonction de l'individu qui la conçoit :

L'unité de traduction est un élément constituant d'un tout qui a sa source, ou base formelle, dans le texte de départ, son aboutissement dans le texte d'arrivée, et qui passe pour sa réalisation par le cerveau du traducteur.

La subjectivité du traducteur et les aspects cognitifs qui sont censés influencer la composition de l'UT ont été notamment pris en compte dans le 
cadre des théories interprétatives de la traduction : à partir de l'observation de l'activité d'interprétation simultanée, Marianne Lederer (2014, p. 41) conçoit des "unités de sens" dont l'ampleur dépend du "moment où apparaît la compréhension". Dans le cadre de la théorie interprétative, on parle de “déverbalisation" pour décrire le phénomène suivant :

Une fois le sens d'un segment de phrase compris, il est libéré de sa forme linguistique, se transforme en unité cognitive que la mémoire retient en tant que telle pendant quelques secondes; l'interprète peut alors la reformuler dans n'importe quelle autre langue sans être esclave des mots de l'original. (Lederer 2018, p. 39)

Ce tournant cognitif a aussi eu le mérite de situer l'opération de traduction sur un plan discursif et non plus seulement linguistique, en inspirant des travaux successifs centrés sur l'importance de la dimension discursive en traductologie.

À l'encontre de l'UT, dont le découpage est prédéterminé, l'unité de sens propose un découpage qui varie par rapport à chaque traducteur, ce qui favorise l'expression de sa créativité (Lederer 2018, p. 42). Comme nous le verrons plus loin, ces deux postures traductives sont toutes les deux impliquées dans la traduction de la chanson.

\subsection{Les niveaux d'analyse d'une chanson}

Avant d'entrer dans le vif du discours, une autre précision s'impose visant à mieux cerner les niveaux d'analyse $d^{\prime}$ une chanson.

Dans le cadre de son approche sémiologique de la chanson, Louis-Jean Calvet (1995, p. 17) se concentre sur "la chanson telle qu' on l'entend, ou telle que 
le disque la transmet, laissant de côté la chanson telle qu'on la voit, sur scène ou sur un écran". Dans le but de comprendre ce qui contribue à la construction du sens d'une chanson, il distingue la "chanson écrite" de la "chanson chantée" : la première, assimilable à la partition, est conçue comme "une sorte de structure abstraite qui est à la 'chanson chantée' ce qu'une notation phonologique est à la réalisation phonétique d'un texte" (Calvet 1995, p. 18). D'après Calvet, les différentes interprétations de la même chanson seraient des variantes derrière lesquelles se cacherait l'invariant constitué par les rapports entre le texte, le rythme et la mélodie. Puisque nos observations linguistiques et traductologiques portent prioritairement sur des éléments structuraux, nous nous focaliserons sur cet invariant, qui représente un objet d'étude suffisamment stable.

En effet, si l'on considère la traduction d'une chanson comme une de ses interprétations possibles (Conenna 1987, p. 100), on s'aperçoit qu'il s'agit d'une version qui comporte des variantes aussi bien sur le plan de la forme que sur celui du contenu ${ }^{5}$. C'est donc à plus forte raison que la chanson écrite de départ peut représenter un bon point de repère pour observer, analyser et décrire les traductions chantées.

$\mathrm{Au}$ besoin, nous prendrons aussi en considération les paroles des chansons : en effet, quoique leur mise en page soit dépourvue de toute référence à la musique, elles ont l'avantage de contenir le texte en entier et de "fournir souvent de manière indirecte - des informations intéressantes et

\footnotetext{
${ }^{5}$ Le principe d'après lequel dans la traduction d'un texte chanté c'est la partition qu'il faut traduire a été déjà formulé par Castil Blaze (1858, p. 127).
} 
complémentaires, comme celles qui témoignent des tentatives de transcrire certains phénomènes de l'oralité" (D'Andrea 2016, p. 57).

\subsection{Quelles unités pour la traduction chantée?}

Une fois admis que la traduction chantée ne peut se passer de la composante musicale, il est question de savoir si et comment on pourrait établir des unités de traduction chantée, autrement dit s'il existe des critères spécifiques pour les identifier.

Lorsqu'on cherche à décrire une traduction de chanson, il faut tenir compte de la multiplicité de signifiants qui la composent, ce qui complique davantage l'identification des unités d'analyse pertinentes. Restons-en au niveau structural, et rappelons la définition de chanson comme "un objet composite qui résulte de la mise en correspondance entre un objet linguistique, le texte, et un objet musical, l'air" (Dell 2003, p. 515). Même si François Dell se réfère à un répertoire vocal relativement restreint, nous adoptons sa proposition terminologique, qui semble largement applicable à la chanson relevant de répertoires différents : il propose de découper le texte en des unités linguistiques appelées "vers" et de découper l'air en des unités musicales appelées "motifs". Selon ses termes, "l'objet composite qui naît de l'association d'un vers et d'un motif" sera appelé "segment" (Dell 1989, p. 124).

Quelles sont les implications d'un tel découpage pour l'analyse des chansons traduites? Nous avons pris en considération des chansons d'auteur et nous les avons comparées à leurs traductions chantées. Ne pouvant pas observer les processus à l'œuvre dans l'opération traduisante, nous nous sommes 
focalisée a posteriori sur ses résultats : la traduction en tant que produit fait donc l'objet de notre étude.

Nous posons d'abord l'hypothèse qu'en principe, dans le passage traductif d'une chanson de départ à une chanson d'arrivée, les vers d'arrivée remplacent les vers de départ et, en se mariant avec les mêmes motifs, donnent lieu aux nouveaux segments d'arrivée.

Et pourtant, dans la réalité des faits, cette schématisation se trouve confrontée à des difficultés : prenons par exemple la célèbre chanson de Georges Brassens Le gorille et la version italienne réalisée par Fabrizio De André. Pour effectuer une comparaison entre les vers de départ et les vers d'arrivée, nous avons mis en regard les paroles de la chanson française et celles de la chanson italienne et nous les avons analysées en tenant compte aussi de leur mise en musique. Focalisons-nous sur la première strophe (Tableau 1).

\section{Tableau 1}

Le Gorille/Il Gorilla

\begin{tabular}{|l|l|l|l|}
\hline VdD & \multicolumn{1}{|c|}{ Brassens } & \multicolumn{1}{c|}{ De André } & VdA \\
\hline 1 & C'est à travers de larges grilles & Sulla piazza d'una città & 1 \\
\hline 2 & Que les femelles du canton & La gente guardava con ammirazione & 2 \\
\hline 3 & Contemplaient un puissant gorille & Un gorilla portato là & 3 \\
\hline 4 & Sans souci du qu'en-dira-t-on & Dagli zingari di un baraccone & 4 \\
\hline 5 & Avec impudeur, ces commères & Con poco senso del pudore & 5 \\
\hline 6 & Lorgnaient même un endroit précis & Le comari di quel rione & 6 \\
\hline 7 & Que, rigoureusement ma mère & Contemplavano l'animale & 7 \\
\hline 8 & M'a défendu d' nommer ici & Non dico come, non dico dove & 8 \\
\hline 9 & Gare au gorille !... & Attenti al gorilla! & 9 \\
\hline
\end{tabular}

Mise en regard des paroles de chanson de la première strophe. 
La strophe italienne peut à raison être globalement considérée comme une traduction de la strophe française, jusqu'à l'avertissement condensé dans le célèbre refrain intégré "Gare au gorille !"/“Attenti al gorilla” : à ce propos, nous pouvons évoquer la technique cinématographique du fondu enchaîné, adoptée par Conenna (2000, p. 153) pour décrire l'image de la chanson originale, qui laisse la place à ses nouvelles versions, dans d'autres langues, tout en transparaissant à travers celles-ci. Malgré les omissions ("de larges grilles", "puissant", "précis", ...) et les ajouts ("Sulla piazza d'una città", "dagli zingari di un baraccone", ...), la version italienne remplit la fonction introductive de la strophe originale : présentation de l'intrigue racontée et du protagoniste, renvoi à l'absence de pudeur des regards des commères et allusion à la censure imposée au "canteur", ce dernier terme étant employé au sens établi par Stéphane Hirschi (2008, p. 20, n. 4) :

\footnotetext{
'Canteur', notion opératoire en cantologie pour désigner dans une chanson l'équivalent du narrateur dans un roman. Personnage ou point de vue, il convient de le distinguer du chanteur, à savoir l'interprète, qui, lui, prête son corps et sa voix le temps d'une chanson, et endosse un nouveau rôle de canteur au morceau suivant.
}

Néanmoins, si l'on se focalise sur les vers parallèles qui résultent de la mise en regard, on constate que le vers d'arrivée 1 n'est pas la traduction du vers de départ 1, que le vers d'arrivée 2 n'est pas la traduction du vers de départ 2, et ainsi de suite. Ce manque d'équivalence sémantique vers à vers ne doit pourtant pas étonner, car c'est un acquis du débat séculaire en matière de littéralisme en traduction, comme le montre déjà la troisième règle formulée par Etienne Dolet (1540, p. 13) : 
Je ne veulx taire ici la follie d'aulcuns traducteurs, lesquels au lieu de liberté se submettent à servitude. C'est à scavoir qu'ils sont si sots, qu'ils s'efforcent de rendre ligne pour ligne, ou vers pour vers. Par laquelle erreur ils dépravent souvent le sens de l'autheur qu'ils traduisent, et n'expriment la grace, et parfection de l'une, et l'aultre langue. Tu te garderas diligemment de ce vice qui ne démonstre aultre chose que l'ignorance du traducteur.

Faut-il en conclure que le découpage en vers des paroles de chanson n'est pas du tout pertinent pour réfléchir aux unités de traduction chantée ? Avant d'établir le constat que les vers parallèles issus de la mise en regard des paroles de chanson ne sont pas nécessairement la traduction l'un de l'autre, il est légitime de se demander ce qu'ils ont en commun. La réponse est aussi simple qu'évidente : ils sont généralement chantés sur le même motif, ce qui nous conduit aussi à approfondir le rôle de la musique dans la traduction chantée.

Loin de représenter seulement une "contrainte", terme qui implique une certaine connotation négative, la musique fait partie de l'invariant qui permet de créer une nouvelle version de la chanson de départ ${ }^{6}$. Du point de vue de la réception, la musique permet de reconnaître, de prime abord, si une chanson donnée est la version d'une autre chanson dans une langue différente. Par des écoutes successives, il sera possible de classer cette version comme une libre adaptation ou comme une véritable traduction. Autrement dit, selon une démarche plus proprement analytique, le traductologue pourra établir s'il s'agit d'une chanson qui ne respecte que la musique originale ou d'une chanson visant à garder un certain degré de fidélité sémantique par rapport au texte de départ.

\footnotetext{
${ }^{6}$ Pour simplifier quelque peu, nous parlons ici d'invariant, tout en sachant que de tout petites variations sont possibles même au niveau musical. Du reste, il s'agit du même genre de variations qui concernent parfois le niveau intralinguistique, d'une strophe à l'autre de la même chanson.
} 
Dans une autre étude ( $D^{\prime}$ Andrea 2014), nous comparons l'acte de traduction d'une chanson au travail créatif du parolier : en somme, au lieu d'être considérée comme une traduction poétique ayant la contrainte supplémentaire de la musique, la traduction d'une chanson serait à interpréter comme la création d'un texte chantable sur une musique donnée et ayant une contrainte sémantique.

Cette perspective met en jeu un critère incontournable en matière de traduction chantée : la chantabilité. Dans le prochain paragraphe, nous verrons comment la prise en compte de ce paramètre, en plus de la notion traditionnelle de fidélité, se répercute sur l'étude des unités de traduction dans la chanson traduite.

\section{Les unités de traduction chantée entre fidélité et chantabilité}

En matière de traduction, la notion de fidélité a été largement débattue et a beaucoup évolué au fil des siècles jusqu'à être reprise, entre autres, par Ladmiral (2014). Dans sa réflexion sur le littéralisme en traduction, il montre comment “entre sourciers et ciblistes, l'opposition n'est pas entre une fidélité plus ou moins grande, mais entre deux modes de fidélité" (p. 18).

Notre propos est ici de nous demander à quoi devrait être fidèle une chanson traduite. Si l'on considère que la chanson est un objet sémiotiquement complexe, résultant de la superposition d'un objet linguistique et d'un objet musical, il s'ensuit que si fidélité est, elle est à rechercher sur ces deux plans? ${ }^{7}$. La

\footnotetext{
${ }^{7}$ Le résultat perceptif de cette superposition va certes bien au-delà de l'addition de ces deux composantes, car il dépend aussi d'autres facteurs tels les différentes interprétations et la subjectivité de chaque auditeur. Néanmoins, nous proposons ici une schématisation correspondant à la perspective structuraliste qui est la nôtre, qui ne tient pas compte de la dimension interprétative de la chanson.
} 
chanson d'arrivée se doit de respecter non seulement le texte source mais aussi sa musique, sans pour autant négliger de respecter "le signifié (ou, plus exactement, le sens et la 'valeur' d'une parole qui doit advenir dans la languecible" (Ladmiral 2014, p. 4).

Dans cette optique, la chanson de départ peut être recréée à travers deux ordres d'unités : d'une part, des unités de sens dont l'ampleur dépend de la subjectivité du traducteur, et d'autre part des unités de traduction dont l'empan coïncide souvent avec celui des vers. Pour ces dernières, il convient de préciser qu'il s'agit de créer de nouveaux vers chantables sur la même musique que celle des vers de départ, à quelques petites variantes près. La chantabilité peut donc être conçue comme étroitement liée à la notion de fidélité.

Illustrons notre propos par un exemple tiré à nouveau de Le Gorille/Il gorilla (Tableau 2) :

Tableau 2

Le Gorille/Il Gorilla

\begin{tabular}{|l|l|l|c|}
\hline 2 & Que les femelles du canton & La gente guardava con ammirazione & 2 \\
\hline
\end{tabular}

Mise en regard du vers 2 de la chanson originale et de la version italienne.

Une fois constaté le manque d'équivalence sémantique entre les vers parallèles ci-dessus, on pourrait s'interroger sur leur structure formelle. Vu les analogies souvent évoquées entre des vers littéraires et des vers de chanson, on pourrait d'abord décrire ces vers parallèles selon des critères métriques, afin de vérifier s'ils ont le même nombre de syllabes pertinentes pour la définition du mètre et de la cadence. En principe, les deux vers ne semblent pas 
interchangeables sur une même musique car ils n'ont ni le même mètre ni la même cadence ${ }^{8}$. En effet, le 8-syllabe à cadence masculine du français est remplacé, en italien, par un 11-syllabe à cadence féminine ${ }^{9}$.

Néanmoins, malgré ces deux points de divergence, le vers d'arrivée est parfaitement chantable, au point qu'il semble avoir été créé en langue cible.

Dans les Tableaux 3 et 4, nous proposons une représentation graphique de l'alignement entre chacun de ces deux vers et la structure musicale de la chanson. Pour ce faire, nous nous inspirons librement des grilles métriques issues de la grammaire générative (Dell et Halle 2009, p. 66) ${ }^{10}$.

\footnotetext{
${ }^{8}$ Remarquons au passage qu'une équivalence en nombre syllabique ne serait pas en soi synonyme d'une parfaite chantabilité du vers d'arrivée, car il faudrait aussi prendre en compte l'agencement des accents et la présence éventuelle de mélismes.

${ }^{9}$ Il n'est peut-être pas inutile de rappeler ici qu'en métrique française les vers sont dénommés sur la base des vers oxytons, alors qu'en métrique italienne leur dénomination se fonde sur des vers paroxytons. Par exemple, un décasyllabe français et un endecasillabo italien, tout en ayant deux dénominations différentes, sont métriquement équivalents car leur dernière voyelle accentuée se trouve en dixième position.

Afin de pouvoir comparer la métrique d'un texte français avec celle d'un texte italien, sans à chaque fois devoir considérer cette différence intrinsèque, nous proposons de dénommer les vers italiens à la manière des vers français, à savoir de prendre en compte les seules syllabes métriques, sans considérer les voyelles surnuméraires. Plus précisément, nous adoptons la terminologie de Benoît de Cornulier (1995, p. 277-278), d'après qui la formule "n-syllabe ou n-voyelle" désigne un vers de n syllabes ou voyelles.

${ }^{10}$ Bien que notre recherche ne se situe pas dans le cadre de la métrique générative, nous avons voulu adopter ce genre de grilles car elles peuvent servir au but qui est le nôtre, en ce qu'elles permettent d'illustrer la manière dont les paroles d'une chanson sont associées à ses instants métriques; en outre, ce genre de grilles a l'avantage de pouvoir être décodé de façon très intuitive par rapport à une partition de musique, qui n'est pas lisible par tout un chacun.
} 
Tableau 3

Le Gorille, v. 2

\begin{tabular}{|c|c|c|c|c|c|c|c|c|c|c|c|c|c|c|}
\hline$x$ & & & & & & $x$ & & & & & & $x$ & & \\
\hline$x$ & & & $x$ & & & $x$ & & & $x$ & & & $x$ & & \\
\hline$x$ & $x$ & $x$ & $x$ & $x$ & $x$ & $x$ & $x$ & $x$ & $x$ & $x$ & $x$ & $x$ & $x$ & $x$ \\
\hline & & & & D & D & D & D & $\mathrm{D}$ & B & $\mathrm{D}$ & C\# & & & \\
\hline & & & & Que & les & fe- & mel- & les & $d u$ & can- & ton & & & \\
\hline
\end{tabular}

Grille métrique du vers 2 de la chanson de départ.

Tableau 4

Il Gorilla, v. 2

\begin{tabular}{|c|c|c|c|c|c|c|c|c|c|c|c|c|c|c|}
\hline $\mathrm{x}$ & & & & & & $\mathrm{x}$ & & & & & & $\mathrm{x}$ & & \\
\hline $\mathrm{x}$ & & & $\mathrm{x}$ & & & $\mathrm{x}$ & & & $\mathrm{x}$ & & & $\mathrm{x}$ & & \\
\hline $\mathrm{x}$ & $\mathrm{x}$ & $\mathrm{x}$ & $\mathrm{x}$ & $\mathrm{x}$ & $\mathrm{x}$ & $\mathrm{x}$ & $\mathrm{x}$ & $\mathrm{x}$ & $\mathrm{x}$ & $\mathrm{x}$ & $\mathrm{x}$ & $\mathrm{x}$ & $\mathrm{x}$ & $\mathrm{x}$ \\
\hline & & $\mathrm{D}$ & $\mathrm{D}$ & $\mathrm{D}$ & $\mathrm{D}$ & $\mathrm{D}$ & $\mathrm{D}$ & $\mathrm{D}$ & $\mathrm{D}$ & $\mathrm{B}$ & $\mathrm{D}$ & $\mathrm{C \#}$ & $\mathrm{C \#}$ & \\
\hline & & $L a$ & gen- & te & guar- & $d a-$ & $v a$ & con & am- & mi- & ra- & zio- & ne & \\
\hline
\end{tabular}

Grille métrique du vers 2 de la chanson d'arrivée.

Ici, les lignes forment un système de référence dans lequel est représentée la disposition dans le temps des notes (indiquées sur la quatrième ligne selon la notation alphabétique) et des syllabes qui y sont associées (indiquées sur la cinquième ligne) ; les " $\mathrm{x}$ " sur la même ligne se trouvent à des intervalles de temps qui sont perçus comme égaux ; les positions métriques sont représentées par les colonnes, dont la hauteur varie en fonction du degré de proéminence métrique. 
Dans le Tableau 5, nous tentons de superposer les données ci-dessus, sans tenir compte de la mélodie :

Tableau 5

Le Gorille/Il Gorilla, v. 2

\begin{tabular}{|l|l|l|l|l|l|l|l|l|l|l|l|l|l|l|}
\hline $\mathrm{x}$ & & & & & & $\mathrm{x}$ & & & & & & $\mathrm{x}$ & & \\
\hline $\mathrm{x}$ & & & $\mathrm{x}$ & & & $\mathrm{x}$ & & & $\mathrm{x}$ & & & $\mathrm{x}$ & & \\
\hline $\mathrm{x}$ & $\mathrm{x}$ & $\mathrm{x}$ & $\mathrm{x}$ & $\mathrm{x}$ & $\mathrm{x}$ & $\mathrm{x}$ & $\mathrm{x}$ & $\mathrm{x}$ & $\mathrm{x}$ & $\mathrm{x}$ & $\mathrm{x}$ & $\mathrm{x}$ & $\mathrm{x}$ & $\mathrm{x}$ \\
\hline & & & & Que & les & fe- & mel & les & $\mathrm{du}$ & can & ton & & & \\
\hline & & La & gen & te & guar & da & va & con & am & mi- & ra- & zio & ne & \\
\hline
\end{tabular}

Grille métrique du vers 2 de la chanson de départ et de la chanson d'arrivée.

Précisons tout de suite que l'alignement proposé dans le Tableau 5 n'est que virtuel. En effet les deux versions (française et italienne) ne sont pas faites pour être chantées en même temps. Sans avoir recours à des partitions musicales, ce tableau voudrait illustrer de manière simple comment scander sur le même accompagnement deux vers ayant un nombre différent de syllabes et un motif partiellement modifié.

En effet, les syllabes supplémentaires (ici en gras) sont chantées sur des notes ajoutées à la mélodie originale mais compatibles avec la structure musicale de la chanson ${ }^{11}$.

\footnotetext{
${ }^{11} \mathrm{D}$ 'ailleurs, ce sont des modifications qui pourraient même concerner le niveau intralinguistique. Il arrive parfois qu'à l'intérieur de la même chanson, d'une strophe à l'autre, des vers chantés sur la même musique n'aient pas le même nombre de syllabes. Néanmoins, cette différence n'est pas forcément perçue à l'écoute.
} 
Ce genre de différences entre une chanson de départ et une chanson d'arrivée, qui n'affectent pas l'invariant de la chanson, se fondent sur de petits ajustements mélodiques dont la description formelle dépasserait les limites de cette contribution et notamment celles de notre domaine disciplinaire. Ici, nous nous contentons de dire qu'en matière de traduction chantée, la musique n'est pas forcément une contrainte au sens strict, mais qu'elle peut aussi bel et bien représenter, dans une certaine mesure, une ressource dont la flexibilité élargit les marges de manœuvre du traducteur ${ }^{12}$. Autrement dit, la musique, en tant que langage, reste susceptible d'être elle-aussi traduite, au sens étymologique du verbe latin traducěre, c'est-à-dire "faire passer".

Nous avons voulu montrer qu'en matière de traduction chantée, la notion de chantabilité ne s'oppose pas à celle de fidélité, elle est une forme de fidélité qui réside dans la compatibilité entre texte d'arrivée et musique de départ. Autrement dit, il s'agit d'un facteur qui contribue largement à atteindre une fidélité plus complète. Quant à la notion traditionnelle d'unité de traduction, fondée sur un découpage du texte à traduire, elle ne s'applique pas aux paroles de chanson, pour lesquelles la seule UT qui semble fonctionner est la strophe, voire le texte entier. À la limite, la notion de découpage semble avoir des chances de fonctionner pour la composante musicale, qui joue un rôle central dans l'établissement de la chantabilité des vers d'arrivée.

\footnotetext{
${ }^{12}$ Celui-ci, bien évidemment, doit avoir des compétences dans le domaine musical ou, du moins, il doit être capable de fredonner une chanson et de juger de la chantabilité de ses solutions traductives, mais cela est une autre histoire...
} 


\section{Conclusions et perspectives}

Dans cette étude, à travers la question des unités de traduction chantée, nous avons davantage réfléchi aux enjeux généraux de la traduction de la chanson. En effet, lorsqu'on essaye d'identifier les unités de traduction d'une chanson, on se heurte essentiellement à des difficultés qui concernent les deux catégories suivantes: d'une part, des unités de sens dont l'ampleur varie au cas par cas ; d'autre part, des vers parallèles dont le sens n'est pas forcément équivalent mais qui doivent pouvoir se chanter à peu près sur la même musique. Nous avons proposé l'hypothèse que ces niveaux sont tous les deux essentiels pour parler de traduction chantée, car la traduction d'une chanson ne saurait se réduire à une traduction de ses paroles. La musique doit aussi être traduite, lorsqu'on vise une traduction chantable.

Pour reprendre la question concernant les unités de traduction chantée, cette recherche a mis en évidence la nécessité de mieux cerner la notion de vers de chanson, car il nous semble qu'au-delà de la dimension typographique, il s'agit d'une notion-clé pour mieux étudier l'interface langue-musique en vue d'une meilleure définition du paramètre appelé "chantabilité".

\section{Références}

BALLARD, Michel. À propos des procédés de traduction. Palimpsestes [En ligne], Hors série, 2006, mis en ligne le 01 septembre 2008. URL: http://journals.openedition.org/palimpsestes/386. Consulté le 10 novembre 2019.

CALVET, Louis-Jean. Approche sémiologique de quelques chansons de Georges Brassens. Études littéraires v. 27, n. 3, p. 17-27, 1995.

CASTIL-BLAZE. De l'opéra en France, vol. 2. Paris: Janet et Cotelle, 1820. 
Des traductions d'opéras. In: L'Art des vers lyriques. Paris: Adolphe Delahays, 1858, p. 127-159.

CONENNA, Mirella. Traduire la chanson: les interprétations italiennes de Georges Brassens. Le Français dans le Monde. Recherches et Applications, numéro special, p. 99-106, 1987.

(Ed.). Georges Brassens. Lingua, poesia, interpretazioni. Fasano: Schena Editore, 1998.

. Dissolvenze incrociate: canzoni e traduzioni di Brassens. In: GARZONE, Giuliana ; SCHENA, Leandro (Eds.). Tradurre la canzone d'autore. Bologna: Clueb, 2000, p. 153-165.

CORNULIER, Benoît de. Art poëtique. Notions et problèmes de métrique. Presses Universitaires de Lyon, 1995.

D'ANDREA, Giulia. Stratégies métriques et traduction des textes chantés. In: WOJCIECHOWSKA, Barbara (Ed.). De la musique avant toute chose. Notes linguistiques et littéraires. Paris: L'Harmattan, 2014, p. 71-85.

. Code graphique et code phonique dans le chant. In BUCHI, Éva et al. (Eds.). Actes du XXVIIe Congrès international de linguistique et de philologie romanes (Nancy, 15-20 juillet 2013), Bibliothèque de linguistique Romane, 14,2. Strasbourg: ÉliPhi, 2016, p. 1047-1057.

DE ANGELIS, Enrico; SACCHI, Sergio Secondiano (Eds.) La Tradotta. Storie di canzoni amate e tradite. Civitella in Val di Chiana: Editrice Zona, 2003.

DELL, François. Concordances rythmiques entre la musique et les paroles dans le chant. L'accent et l'e muet dans la chanson française. In: DOMINICY, Marc (Ed.) Le souci des apparences. Éditions de l’Université de Bruxelles, 1989, p. 121-136.

. Répétitions parallèles dans les paroles et dans la musique des chansons. In: AROUI, Jean-Louis (Ed.) Le sens et la mesure. De la pragmatique à la métrique. Hommages à Benoît de Cornulier. Paris: Champion, 2003, p. 499-522. DELL, François; HALLE, John. Comparing Musical Textsetting in French and in English songs. In: ARLEO, Andy; AROUI, Jean-Louis (Eds.). Towards a 
Typology of Poetic Forms. From language to metrics and beyond. Amsterdam: John Benjamins Publishing Company, 2009, p. 63-78.

DOLET, Etienne. De la maniere de bien traduire d'une langue en aultre. Lyon: Etienne Dolet, 1540.

FRIGAU, Céline. Écriture, édition, traductions à lire et à chanter : les activités multiples de Manfredo Maggioni, staff librettist à Londres. In: DECROISETTE, Françoise (Ed.) Le livret d'opéra, œuvre littéraire? Saint-Denis: Presses Universitaires de Vincennes, 2011, p. 87-124.

GARZONE, Giuliana; SCHENA, Leandro (Eds.) Tradurre la canzone d'autore. Bologna: Clueb, 2000.

GARZONE, Giuliana. La canzone di Bob Dylan in Italia: traduzione e appropriazione. In: GARZONE, Giuliana; SCHENA, Leandro (Eds.). Tradurre la canzone d'autore. Bologna: Clueb, 2000, p. 77-105.

HIRSCHI, Stéphane. Chanson. L'art de fixer l'air du temps. De Béranger à Mano Solo. Paris: Les Belles Lettres, 2008.

LADMIRAL, Jean-René. L'Esthétique de la traduction et ses prémisses musicales. In: MARSCHALL, Gottfried (Ed.) La traduction des livrets. Aspects théoriques, historiques et pragmatiques. Presses de l'Université ParisSorbonne, 2004, p. 29-41.

Sourciers et ciblistes. In: Sourcier ou cibliste. Les profondeurs de la traduction. Paris: Les Belles Lettres, 2014, p. 3-27.

LEDERER, Marianne. Implicite et explicite. In : SELESKOVITCH, Danica; LEDERER, Marianne (Eds.) Interpréter pour traduire, nouvelle édition revue et corrigée. Paris: Les Belles Lettres, 2014, p. 39-85.

. Unité de traduction ou unité de sens ? Du linguistique au cognitive.

Équivalences 45, p. 27-44, 2018.

LOW, Peter. The Pentathlon Approach to Translating Songs. In: GORLÉE, Dinda L. (Ed.). Song and Significance. Virtues and Vices of Vocal Translation. Amsterdam: Rodopi, 2005, p. 185-212.

MARSCHALL, Gottfried R. (Ed.) La traduction des livrets. Aspects théoriques, historiques et pragmatiques. Presses de l’Université Paris-Sorbonne, 2004. 
MENIN, Roberto. Bertold Brecht, la canzone a teatro, l'Opera da tre soldi. In: GARZONE, Giuliana; SCHENA, Leandro (Eds.). Tradurre la canzone d'autore. Bologna: Clueb, 2000, p. 45-63.

SACCHI, Sergio S. Traduttori \& Traditori. In: DE ANGELIS, Enrico; SACCHI, Sergio S. (Eds.). La Tradotta. Storie di canzoni amate e tradite. Civitella in Val di Chiana: Editrice Zona, 2003, p. 8-20.

SALMON KOVARSKY, Laura. Criteri e opzioni per tradurre canzoni. A proposito di Vysockij in italiano. In: GARZONE, Giuliana; \& SCHENA, Leandro (Eds.) Tradurre la canzone d'autore. Bologna: Clueb, 2000, p. 115-133.

SCHNEIDER, Corinne. La critique parisienne et la question de l'opéra traduit (1820-1870). In: TRIAIRE, Sylvie; BRUNET, François (Eds.) Aspects de la critique musicale au XIXe siècle. Montpellier: Presses Universitaires de la Méditerranée, 2002, p. 31-46. URL: http://books.openedition.org/pulm/251?lang=fr. Consulté le 21 novembre 2019. SUSAM-SARAJEVA, Sebnem. Translation and Music. Changing Perspectives, Frameworks and Significance. The Translator v. 14, n. 2, p. 187-200, 2008. VINAY, Jean-Paul; DARBELNET, Jean. Stylistique comparée du français et de l'anglais. Paris: Les Éditions Didier, 1958.

\section{Résumé}

Cet article a pour but d'explorer quelques spécificités de la traduction chantée domaine indûment négligé en traductologie - en réfléchissant notamment à la pertinence de la notion d'unité de traduction. Après avoir distingué la traduction chantée proprement dite des autres formes de traductions de textes chantés, ainsi que des adaptations beaucoup plus libres, nous proposons un rapide tour d'horizon contenant un état de l'art des études sur ce sujet. Ensuite, nous nous focalisons sur la notion d'unité de traduction pour essayer enfin d'en évaluer une possible application aux éléments structuraux de la chanson.

Mots-clés: Traduction chantée; Unité de traduction; Chantabilité; Chanson. 


\begin{abstract}
This paper aims to analyse the features of singing translation - a highly neglected area of research within Translation Studies - by focusing on the concept of the translation unit. Sung texts may be translated for different purposes, such as singing the target text or simply understanding the source text. The features of singing translation are described and compared to those of much freer adaptations. A brief overview of the state of the art of singing translation is also provided. The analysis pursued in this paper shows that the concept of singability plays a relevant role in achieving a higher degree of fidelity.
\end{abstract}

Keywords: Singing translation; Translation unit; Singability; Song. 